\title{
Acute Inflammatory Infiltrate
}

National Cancer Institute

\section{Source}

National Cancer Institute. Acute Inflammatory Infiltrate. NCI Thesaurus. Code C35979.

A morphologic finding indicating the presence of neutrophils and/or eosinophils in a tissue sample. 